\title{
Management of intercondylar fractures of the distal humerus in adults using bi-columnar plating through trans-olecranon approach
}

\author{
Vijendra Yadav ${ }^{1}$, Parvez Ahmad Ganie ${ }^{2}$, Rajendra Pranav Prasad ${ }^{3}$, Madhan Jeyaraman ${ }^{1 *}$
}

\author{
${ }^{1}$ Department of Orthopaedics, Citi Hospital, Bengaluru, Karnataka, India \\ ${ }^{2}$ Department of Orthopaedics, School of Medical Sciences and Research, Sharda University, Greater Noida, Uttar \\ Pradesh, India \\ ${ }^{3}$ Department of Orthopaedics, JJM Medical College, Davangere, Karnataka, India
}

Received: 28 July 2021

Accepted: 18 September 2021

\author{
*Correspondence: \\ Dr. Madhan Jeyaraman, \\ E-mail: madhanjeyaraman@gmail.com
}

Copyright: (c) the author(s), publisher and licensee Medip Academy. This is an open-access article distributed under the terms of the Creative Commons Attribution Non-Commercial License, which permits unrestricted non-commercial use, distribution, and reproduction in any medium, provided the original work is properly cited.

\begin{abstract}
Background: Inter-condylar fractures of the distal humerus are uncommon injuries and present the most difficult challenge among fractures of the lower end of the humerus. The principle involves anatomical reduction and fixation with stabilization of extra-osseous implants. The functional outcome has been greatly influenced by the early graduated rehabilitative exercise. We aimed to evaluate the functional outcome of inter-condylar fractures of the distal humerus in adults using bi-columnar plating through a trans-olecranon approach.

Methods: A prospective study with 40 adult patients with inter-condylar fracture of the distal humerus were managed with bi-columnar plating through a trans-olecranon approach and were followed up for 12 months. The functional outcome was analysed by Mayo's Elbow Performance Score (MEPS) at the end of 12 months follow up.

Results: The average age of study participants were 50.8 years. A total of 24 cases were due to RTA and 16 cases were due to direct falls. Out of 40 cases, $6(15 \%)$ were of B1 type, $2(5 \%)$ were of type B2, $12(30 \%)$ were of C1, $10(25 \%)$ of $\mathrm{C} 2$, and $10(25 \%)$ were of the type of $\mathrm{C} 3$ according to AO classification. According to MEPS at the end of the 12thmonth follow-up, excellent results were seen in $24(60 \%)$, good in $8(20 \%)$, fair in $6(15 \%)$, and poor results in $2(5 \%)$ cases.

Conclusions: Anatomically, bi-columnar plating for distal humerus is useful in providing stable fixation for complex distal articular fractured by trans-olecranon approach and facilitating early postoperative rehabilitation. Clinical and radiological results showed a good healing rate with a good range of motion.
\end{abstract}

Keywords: Distal humerus, Inter-condylar, Bi-columnar plating, Trans-olecranon

\section{INTRODUCTION}

Approximately $7 \%$ of all adult fractures involve the elbow, of these one-third involve the distal humerus. ${ }^{1}$ Distal humerus fractures, therefore comprise around $2 \%$ of all fractures. ${ }^{2,3}$ Distal humerus fractures remain one of the most challenging injuries to manage. ${ }^{4}$ They are commonly multi-fragmented, occur in osteopenia bone, and have complex anatomy with limited options for internal fixation.
Treatment outcomes are often associated with elbow stiffness, weakness, and pain. A painless, stable, and mobile elbow joint is desired as it allows the hand to conduct the activities of daily living. Depending upon the comminution and displacement, open reduction and internal fixation with $1 / 3$ tubular plate, pre-contoured LCP, reconstruction plate, Kirschner wire, and double tension band wiring can be done individually or in combination. ${ }^{7-9}$ The result of operative fixation of fractures of the distal humerus remained unpredictable until improved techniques for the fixation of small, articular 
fractures as developed by the Arbeitsgemeinschaft fur Osteosynthesefragen/association for the study of internal fixation (AO/ASIF) and others were applied. ${ }^{10,11}$

With the available literature, fixation with two plates at 90 degrees angle with one another has become the standard of care. ${ }^{12,13}$ Despite the confidence in surgical fixation of distal humerus fractures, these fractures remain challenging to treat effectively by surgeons with interest and experience in skeletal trauma involving the upper extremity. A surgeon treating a healthy active patient with a fracture of the distal humerus should make every attempt to reconstruct and preserve the distal humerus. ${ }^{14,15}$ The quality of elbow function following inter-condylar fractures is related to the degree to which normal anatomic relationships are restored. Residual elbow stiffness remains the worst complication of inter-condylar fractures as it is poorly tolerated because of the lack of compensatory motions in adjacent joints. ${ }^{13}$

We aimed to evaluate the functional outcome of surgical management of intercondylar fracture of the distal humerus in adults by bi-columnar plating through the trans-olecranon approach.

\section{METHODS}

After getting IEC approval, we recruited 40 cases of intercondylar fracture of the distal humerus from July 2016 to June 2019 satisfying the defined inclusion and exclusion criteria. Patients age above 18 years and with intercondylar fracture of the distal end of the humerus have been included in the study. Patients with compound fractures of the distal humerus and old fractures of the distal humerus have been excluded from the study.

A thorough clinic-radiological examination was performed before the surgical management. CT scan of the distal humerus was performed to study the fracture pattern, comminution of fragments, and articular congruity. All cases were treated surgically with orthogonal bi-columnar plating construct to reconstruct the fracture fragments. All the patients were followed up at the end of 1, 3, 6, and 12 months with radiological examination and functional evaluation with Mayo Elbow Performance Score (MEPS).

The descriptive statistics were reported as mean (SD) for continuous variables, frequencies (percentage) for categorical variables. Data were statistically evaluated with IBM Statistical package for social sciences (SPSS) Statistics for windows, version 25.0, IBM Corp, Chicago, IL. The p-value of less than 0.05 was considered significant.

\section{RESULTS}

A total of 40 cases of intercondylar fractures of the distal humerus were treated by open reduction and internal fixation with a pre-contoured bicolumnar locking compression plate through a trans-olecranon approach.

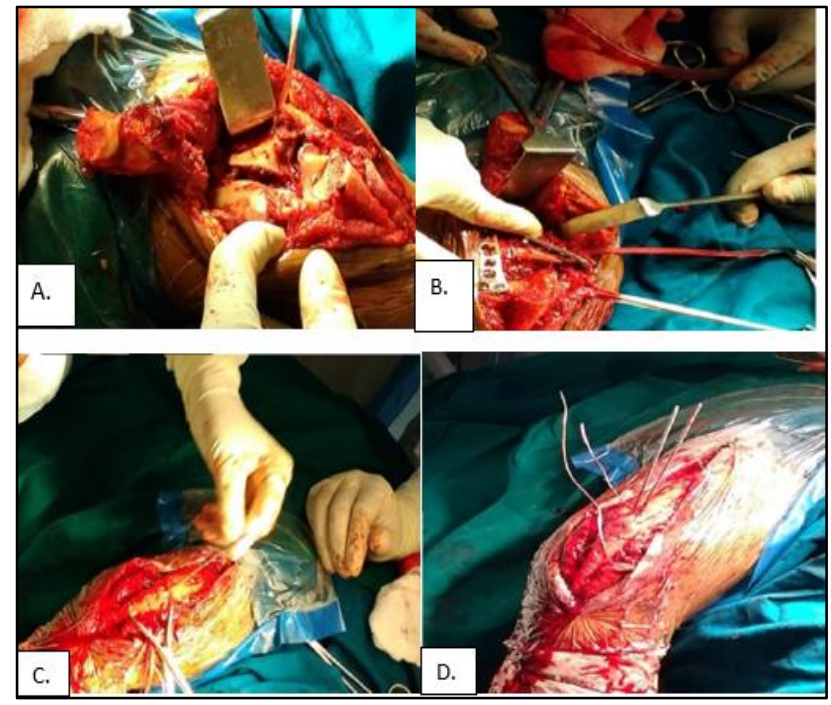

Figure 1: Surgical management of distal humerus with bi-columnar plating through a trans-olecranon

approach. (A) Exposure of fracture site after olecranon osteotomy. (B) Distal humerus fracture reconstructed using bi-columnar platin. (C) Reduction of osteotomized fragments. (D) Tension band wiring of olecranon using $\mathrm{K}$ wires.

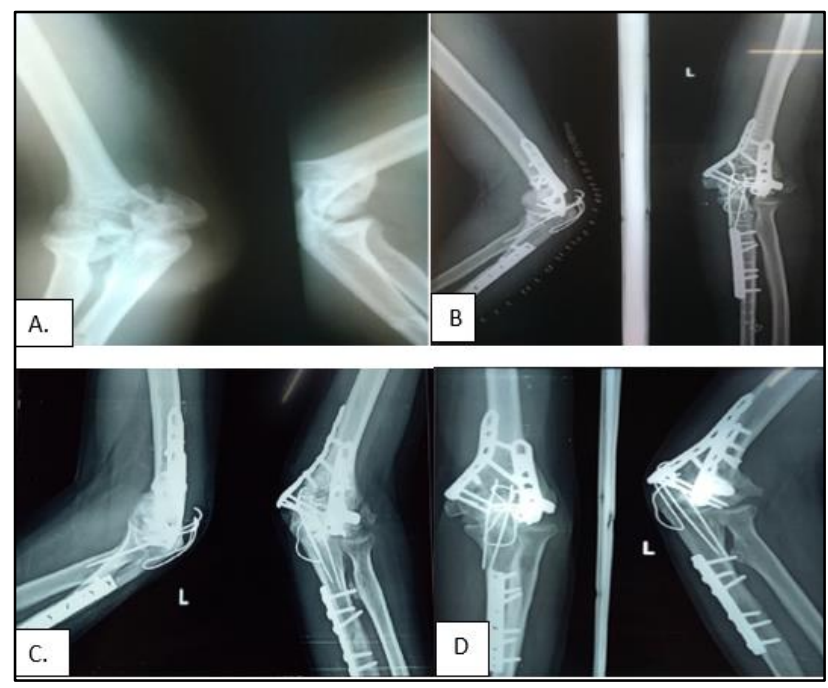

Figure 2: Pre- and post-surgical radiographs of left distal humerus fracture with left proximal ulnar fracture. (A) Preoperative X-ray of left elbow. (B) Immediate post-operative $\mathrm{X}$-ray of the left elbow with bi-columnar plating and proximal ulnar fracture with plating. (C): 3 months follow-up X-ray. (D) 12 months follow-up X-ray.

The range of age was between 22-70 years, with a mean age of 50.8 years. The maximum incidence was in the fourth $(n=14)$ and fifth $(n=8)$ decades. Males $(70 \%)$ outnumbered females $(30 \%)$ in this series. A total of 16 cases $(40 \%)$ were due to direct fall injury and 24 cases $(60 \%)$ were due to road traffic accidents. 


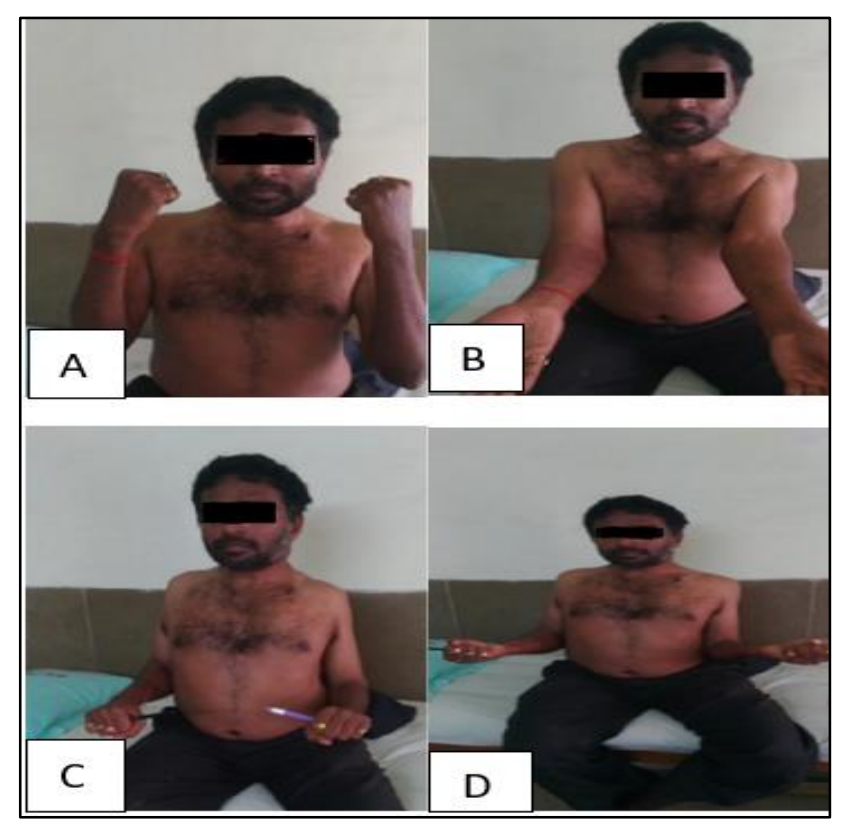

Figure 3: Range of movements at the end of 12 thmonth follow-up. (A) Elbow flexion. (B) Elbow extension. (C) Pronation. (D) Supination.
There were no cases of intraoperative complications. In postoperative period, 5 cases reported complications such as ulnar neuropathy $[(\mathrm{n}=1,2.5 \%)$, spontaneous recovery after 8 weeks], non-union [ $(n=1,2.5 \%)$, treated with bone grafting], superficial infections [ $(n=2,5 \%)$, treated with IV and oral antibiotics according to the culture sensitivity report], and implant failure $[(\mathrm{n}=1,2.5 \%)$, back-out of $6.5 \mathrm{~mm}$ AO cannulated screw in TBW in olecranon osteotomy after one year of surgery in that case TBW was removed]. In the present study, at the end of 12 months, there were 24 cases $(60 \%)$ had excellent results, $8(20 \%)$ had good results, $6(15 \%)$ had fair and $2(5 \%)$ had poor results as per Mayo Elbow Performance scoring system as shown in the table.

In the present series, according to the $\mathrm{AO}$ classification of distal humerus fractures, there were $6(15 \%)$ cases of type B1 fractures, $2(5 \%)$ cases of type B2 fractures, $12(30 \%)$ cases of type $\mathrm{C} 1$ fractures, $10(25 \%)$ cases of $\mathrm{C} 2$ fractures and $10(25 \%)$ cases of type $\mathrm{C} 3$ fractures. Out of 40 cases, 7 cases were reported with associated injuries [1 ulnar \#, 1 distal end radius \#, 1 radial nerve palsy, 1 elbow dislocation, 1 chest trauma, and 2 head injuries] which were addressed and treated appropriately.

Table 1: Functional outcome concerning the type of fracture.

\begin{tabular}{|c|c|c|c|c|c|c|}
\hline \multirow{3}{*}{ Results } & \multicolumn{5}{|c|}{ Type of fractures } & \multirow{2}{*}{ Total } \\
\hline & B1 & B2 & C1 & $\mathrm{C2}$ & C3 & \\
\hline & $\mathbf{N}(\%)$ & $\mathbf{N}(\%)$ & $\mathbf{N}(\%)$ & $\mathbf{N}(\%)$ & $\mathbf{N}(\%)$ & $\mathrm{N}(\%)$ \\
\hline Excellent & $4(66.7)$ & $2(100.0)$ & $10(83.3)$ & $6(60.0)$ & $2(20.0)$ & $24(60.0)$ \\
\hline Good & $2(33.3)$ & - & - & $2(20.0)$ & $4(40.0)$ & $8(20.0)$ \\
\hline Fair & - & - & - & $2(20.0)$ & $4(40.0)$ & $6(15.0)$ \\
\hline Poor & - & - & $2(16.7)$ & - & - & $2(5.0)$ \\
\hline Total & $6(100)$ & $2(100)$ & $12(100)$ & $10(100)$ & $10(100)$ & $40(100)$ \\
\hline
\end{tabular}

\section{DISCUSSION}

Intercondylar fractures of the distal humerus are difficult to treat because of the nature of the injury and the fact that most surgeons do not have a great deal of experience with them. ${ }^{16,17}$ Most intra-articular fractures of the distal humerus are often displaced and therefore the successful treatment demands an anatomic reduction, stable fixation, and the ability to allow early elbow motion. ${ }^{18-20}$ This can be safely achieved by stabilization of fracture fragments with plate osteosynthesis based on restoration of joint congruity.

Previous treatment methods of closed reduction with immobilization, traction, and limited internal fixation have caused significant functional impairment with loss of range of movement. Hence, it is now generally accepted that the most favorable outcome of displaced intraarticular fractures is provided by surgical reconstruction. ${ }^{4}$
Different approaches have been described for type C distal humerus fracture repair. ${ }^{19,21}$ The posterior approach has been used by many surgeons because it exposes the articular surface of the distal humerus sufficiently. ${ }^{22,23}$

Although various approaches have been used for the reduction and fixation of distal humeral fractures, the posterior approach through an olecranon osteotomy is the most widely used. ${ }^{21,24-26}$ This approach provides excellent visualization, particularly of the distal articular fragments and the plate fixation. ${ }^{27-30}$ In this study, a posterior approach with chevron-V-shaped olecranon osteotomy was done in most of the cases. In our study, we did not observe any cases of non-union of olecranon osteotomy. The use of chevron-V osteotomy has decreased the incidence of non-union. All fractures as well as the chevron-V osteotomy united by $10-16$ weeks. 
In this study, we used bi-columnar locking compression plates to reconstruct both the medial and lateral columns as the locking plates provide a fixed plate screw construct with multiple screw options for easy application in distal complex fractures thereby providing angular stability. There is no consensus that whether the orthogonal or parallel plating is superior for fixation. ${ }^{31-33} \mathrm{We}$ used orthogonal plating because it provides better mechanical stability although it requires more extensive soft tissue dissection. The functional outcome was based upon the Mayo Elbow Performance Score which was excellent in 24 patients $(60 \%)$, good in 8 patients $(20 \%)$, fair in 6 patients $(10 \%)$, and poor in 2 patients $(5 \%)$.

\section{CONCLUSION}

Anatomically, bi-columnar plating for distal humerus is useful in providing stable fixation for complex distal articular fractured by trans-olecranon approach and facilitating early postoperative rehabilitation to improve the functional quality of life.

\section{Funding: No funding sources}

Conflict of interest: None declared

Ethical approval: The study was approved by the institutional ethics committee

\section{REFERENCES}

1. Sheehan SE, Dyer GS, Sodickson AD, Patel KI, Khurana B. Traumatic Elbow Injuries: What the Orthopedic Surgeon Wants to Know. RadioGraphics. 2013;33(3):869-88.

2. Amir S, Jannis S, Daniel R. Distal humerus fractures: a review of current therapy concepts. Curr Rev Musculoskelet Med. 2016;9(2):199-206.

3. Fu EC, Ring D. Distal Humerus Fractures. In: Papadakos PJ, Gestring ML, eds. Encyclopedia of Trauma Care. Springer; 2015:476-85.

4. Ul Islam S, Glover AW, Waseem M. Challenges and Solutions in Management of Distal Humerus Fractures. Open Orthop J. 2017;11:1292-307.

5. Youssef B, Youssef S, Ansara S, Porter K. Fractures of the distal humerus. Trauma. 2008;10(2):125-32.

6. Jupiter JB, Neff U, Holzach P, Allgöwer M. Intercondylar fractures of the humerus. An operative approach. J Bone Joint Surg Am. 1985;67(2):226-39.

7. Mahapatra S, Abraham VT. Functional Results of Intercondylar Fractures of the Humerus Fixed with Dual Y-Plate; A Technical Note. Bull Emerg Trauma. 2017;5(1):36-41.

8. Trikha V, Goyal T. Intercondylar humerus fractures: current concepts and controversies. Journal of Clinical Orthopaedics \& Trauma. 2010;1(2):57-65.

9. Chen H, Li D, Zhang J, Xiong X. Comparison of treatments in patients with distal humerus intercondylar fracture: a systematic review and metaanalysis. Annals of Medicine. 2017;49(7):613-25.
10. Lee HJ. Surgical Treatment Strategy for Distal Humerus Intra-articular Fractures. Clin Shoulder Elb. 2019;22(2):113-7.

11. Letsch R, Schmit-Neuerburg KP, Stürmer KM, Walz M. Intraarticular fractures of the distal humerus. Surgical treatment and results. Clin Orthop Relat Res. 1989;(241):238-44.

12. O'Driscoll SW. Optimizing stability in distal humeral fracture fixation. J Shoulder Elbow Surg. 2005;14(1):186S-94S.

13. Savvidou OD, Zampeli F, Koutsouradis P. Complications of open reduction and internal fixation of distal humerus fractures. EFORT Open Rev. 2018;3(10):558-67.

14. Beazley JC, Baraza N, Jordan R, Modi CS. Distal Humeral Fractures-Current Concepts. The Open Orthopaedics Journal. 2017;11(1).

15. Holdsworth BJ, Mossad MM. Fractures of the adult distal humerus. Elbow function after internal fixation. J Bone Joint Surg Br. 1990;72(3):362-5.

16. Pollock JW, Athwal GS, Steinmann SP. Surgical exposures for distal humerus fractures: A review. Clinical Anatomy. 2008;21(8):757-68.

17. Pollock JW, Faber KJ, Athwal GS. Distal humerus fractures. Orthop Clin North Am. 2008;39(2):187200.

18. Anglen J. Distal humerus fractures. J Am Acad Orthop Surg. 2005;13(5):291-7.

19. Hu B, Liu X-W, Huang J-J. [Surgical treatment for distal humerus type $\mathrm{C}$ fractures]. Zhongguo $\mathrm{Gu}$ Shang. 2018;31(10):976-82.

20. Varecka TF, Myeroff C. Distal Humerus Fractures in the Elderly Population. J Am Acad Orthop Surg. 2017;25(10):673-83.

21. Pajarinen J, Björkenheim J-M. Operative treatment of type $\mathrm{C}$ intercondylar fractures of the distal humerus: results after a mean follow-up of 2 years in a series of 18 patients. J Shoulder Elbow Surg. 2002;11(1):48-52.

22. Wu Z-Z, Wang J-D, Ji X-X, Ma Z-J, Wu J-H, Wang Q-G. Surgical exposures of the distal humeral fractures: An anatomical study of the anterior, posterior, medial and lateral approaches. Chin J Traumatol. 2018;21(6):356-9.

23. Mardanpour K, Rahbar M. Open Reduction and Internal Fixation of Intraarticular Fractures of the Humerus: Evaluation of 33 Cases. Trauma Mon. 2013;17(4):396-400.

24. Aslam N, Willett K. Functional outcome following internal fixation of intraarticular fractures of the distal humerus (AO type C). Acta Orthop Belg. 2004;70(2):118-22.

25. Pankaj A, Mallinath G, Malhotra R, Bhan S. Surgical management of intercondylar fractures of the humerus using triceps reflecting anconeus pedicle (TRAP) approach. Indian J Orthop. 2007;41(3):21923.

26. Zalavras CG, Papasoulis E. Intra-articular fractures of the distal humerus-a review of the current practice. Int Orthop. 2018;42(11):2653-62. 
27. Ramsey DC, Thompson AR, Nazir OF, Mirarchi AJ. A new technique for olecranon osteotomy in the treatment of distal humeral fractures. JSES Open Access. 2019;3(1):1-4.

28. Singh R, Singh H, Kanodia N. Olecranon Osteotomy Approach for Complex AO-13C Fractures of Distal Humerus: A Prospective Analysis of 24 Cases. Malays Orthop J. 2019;13(1):30-5.

29. Wilkinson JM, Stanley D. Posterior surgical approaches to the elbow: a comparative anatomic study. J Shoulder Elbow Surg. 2001;10(4):380-2.

30. Wilson ES, Buckwalter JAV, Henning G, Davison J, Fleury IG, Willey MC. Inclusion of Olecranon Osteotomy With the Posterior Approach for Fixation of Distal Humerus Fractures (OTA/AO 13) Does Not Increase Surgical Complications. Journal of Orthopaedic Trauma. 2021;35(7):e223.

31. Atalar AC, Tunalı O, Erşen A, Kapıcıŏglu M, Sağlam Y, Demirhan MS. Biomechanical comparison of orthogonal versus parallel double plating systems in intraarticular distal humerus fractures. Acta Orthopaedica et Traumatologica Turcica. 2017;51(1):23-8.

32. Moon J-G, Lee J-H. Orthogonal versus Parallel Plating for Distal Humeral Fractures. Clinics in Shoulder and Elbow. 2015;18(2):105-2.

33. Zalavras CG, Vercillo MT, Jun B-J, Otarodifard K, Itamura JM, Lee TQ. Biomechanical evaluation of parallel versus orthogonal plate fixation of intraarticular distal humerus fractures. J Shoulder Elbow Surg. 2011;20(1):12-20.

Cite this article as: Yadav V, Ganie PA, Prasad RP, Jeyaraman M. Management of intercondylar fractures of the distal humerus in adults using bicolumnar plating through trans-olecranon approach. Int J Res Orthop 2021;7:1156-60. 